\title{
Asymmetric STDP in excitatory-inhibitory coupled neurons: a self-stabilizing mechanism? Thomas Voegtlin
}

Address: INRIA Lorraine, Campus Scientifique, F-54506 Vandoeuvre les Nancy, France

Email: Thomas Voegtlin - voegtlin@loria.fr

from Eighteenth Annual Computational Neuroscience Meeting: CNS*2009

Berlin, Germany. 18-23 July 2009

Published: I 3 July 2009

BMC Neuroscience 2009, I0(SuppI I):PI39 doi:I0.II86/I47|-2202-I0-SI-PI39

This abstract is available from: http://www.biomedcentral.com/I47I-2202/I0/SI/PI39

(c) 2009 Voegtlin; licensee BioMed Central Ltd.

Spike Timing Dependent Plasticity (STDP), where the modification of a synapse depends on the time difference between pre- and post-synaptic spikes, has been observed in various types of neurons. In general, the STDP window function of excitatory synapses has a causal shape; a causal firing order (where the pre-synaptic neuron fires before the post-synaptic neuron) induces LTP, and an anti-causal firing order induces LTD. In contrast, anti-causal STDP has been described in some inhibitory neurons [1].

In sensory systems, populations of excitatory and inhibitory neurons are often coupled in recurrent excitatoryinhibitory loops. Computational studies have shown that this coupling is the source of beta and gamma-type oscillations. In this type of coupling, excitatory neurons drive the activity of inhibitory neurons and inhibitory neurons in turn gate the activity of excitatory neurons [2]. In this context, we propose to interpret the asymmetric shape of STDP as a self-stabilizing mechanism. We have developed a model of excitatory-inhibitory coupling, where STDP is present at both excitatory and inhibitory synapses [3]. In this model, pre- and post-synaptic spikes are caused by oscillations of the network. Their relative timing depends not only on intrinsic properties of the neurons, but also on the value of synaptic weights, which are controlled by a STDP learning rule.

A fundamental question for computational models of learning is the stability of any learning rule. In our recurrent model, the presence of feedback leads to stability. The mechanism is as follows. When the synaptic weight of an excitatory neuron is increased, this tends to speed up the spike of its post-synaptic inhibitory neuron. This speed-up will in turn delay the next spike of the excitatory neuron, because it will receive inhibition earlier in its firing cycle. Thus, the overall effect of increasing the weight is that the pre-post spike time interval on the next cycle is reduced. In contrast, if the synaptic weight is decreased, then the prepost spike time interval is increased.

This results in the existence of a stable equilibrium, where each synapse receives, on average, the same quantity of LTP and LTD. In this stable regime, the network can be trained to develop a rich repertoire of stable receptive fields [3].

\section{References}

I. Tzounopoulos T, Kim Y, Oertel D, Trussell LO: Cell-specific, spike timing-dependent plasticities in the dorsal cochlear nucleus. Nat Neurosci 2004, 7:71 9-725.

2. Börgers C, Kopell N: Synchronization in networks of excitatory and inhibitory neurons with sparse, random connectivity. Neural Computation 2003, 15:509-538.

3. Voegtlin T: Adaptive synchronization of activities in a recurrent network. Neural Computation 2009 in press. 